\title{
A modified approach for solving a fuzzy multi-objective programming problem
}

\author{
Ahmed M. K. Tarabia ${ }^{1}$, Mohamed A. E. Kassem² ${ }^{2}$ and Noha M. El-Badry ${ }^{1 *}$
}

*Correspondence:

nooha_moh@yahoo.com

${ }^{1}$ Mathematics Department,

Faculty of Science, Damietta

University, Damietta, Egypt

Full list of author information

is available at the end of the article

\begin{abstract}
Based on ranking of fuzzy numbers which deals with fuzzy-valued multi-objective programming problem and the modified crisp model, a modified approach is proposed. Also, two algorithms that play a pivotal role in the proposed method are introduced. The first one returns a ranking function to a given fuzzy number and the second algorithm uses the modified crisp model to deliver a Pareto optimal solution. Moreover, we investigate the stability of the first kind of the solution which is obtained using these algorithms. Finally, a numerical example is given to illustrate our modified approach, using Maple program.
\end{abstract}

Keywords: Multi-objective, Nonlinear programming, Fuzzy number, Ranking function, Scalarization function

\section{Background}

In fuzzy mathematical programming models, all or some of the parameters can be defined as fuzzy numbers. For the models in which all the parameters are defined as fuzzy numbers, there is one important point; how the optimal value of the objective function will be found. Jiménez et al. $(2000,2007)$ said that the answer is related to the ranking of fuzzy numbers. Fuzzy ranking procedure can be used as a part of the solution mechanism for solving fuzzy mathematical programs in which the coefficients of the objective function, coefficients of the constraints and right-hand values of the constraints are defined as fuzzy numbers. Although there are several studies on solving fuzzy mathematical programming problems by employing fuzzy ranking methods, generally, the fuzzy mathematical programming problems are first transformed into classical mathematical programs then solved using conventional techniques in the literature (Rommelfanger 1996).

In general, the nonlinear programming problem to find a solution, which minimizes the objective function under given constraints, one whose objective function and constraints region are convex, is called convex programming problem. For such convex programming problems, there have been proposed many efficient solution methods as the successive quadratic programming method and the general gradient method. Unfortunately, there have not been proposed any decisive solution method for nonconvex programming problems. In recent years, with the diversification of social requirements, the demand for the programs with multiple objective functions, which may be conflicting

(c) The Author(s) 2017. This article is distributed under the terms of the Creative Commons Attribution 4.0 International License (http://creativecommons.org/licenses/by/4.0/), which permits unrestricted use, distribution, and reproduction in any medium, provided you give appropriate credit to the original author(s) and the source, provide a link to the Creative Commons license, and indicate if changes were made. 
with each other, rather than a single-objective function, has been increasing (e.g. maximizing the total profit and minimizing the amount of pollution in a production planning). Since there do not always exist a completely optimal solution which optimizes all objectives simultaneously for multi-objective programming problems, the Pareto optimal solution or non-inferior solution is defined. A solution is Pareto optimal if any improvement of one objective function can be achieved only at the expense of at least one of the other objective functions. For such multi-objective optimization problems, fuzzy programming approaches (e.g. Zimmermann 1983; Rommelfanger 1996), considering the imprecise nature of the DM's judgments in multi-objective optimization problems, seem to be very applicable and promising. The application of the fuzzy set theory at the multi-objective linear programming problems was considered firstly by Dubois and Prade (1980). They stipulating that "When the complexity of system increases, our ability to formulate precise and yet meaningful statement on this system decreases up to a threshold beyond which precision and significance become mutually exclusive characteristics" is also instructive in this regard. This gives substance to the study of mathematical models under uncertainty. As probability theory is a matured segment and a familiar territory of mathematics, it is not a surprise that early works on mathematical programming under uncertainty were devoted to situations where randomness is in the state of affairs (Adeyefa and Luhandjula 2011; Kampempe and Luhandjula 2012; Kall 1976; Vajda 1972). Nevertheless, imprecision cannot be equated with randomness. As a matter of fact, there is a qualitatively different type of imprecision (vagueness) which cannot be tackled with probabilistic apparatus (Zadeh 1978). This has rightly led some researchers to embark upon the investigation of ways of integrating fuzzy relations and/or fuzzy quantities into mathematical programming models (Zimmermann 1987; Inuiguchi and Ramik 2000; Luhandjula 2007).

Our motivation here is to modify an approach that gives a good reflection in reality as well as yields a computationally tractable deterministic problem. Here, a multiobjective programming problem with fuzzy objective functions is considered, which is an ill-defined problem. Neither solution concepts (like Pareto optimality) nor existing approaches (like the weighting method) are introduced for deterministic multi-objective programming and can be blindly applied. On applying the ranking fuzzy numbers tool, it should be properly tailored to take into consideration the fuzziness surrounding the problem. Moreover, existing approaches for solving the above-mentioned problem either caricature the reality or are computationally demanding. The remaining of the paper is as follows. In "Preliminaries" section, we give the brief primer notions of ranking function and fuzzy numbers, and discuss the algorithm for giving fuzzy numbers. In "Finding a satisfying solution of a multi-objective program with fuzzy objective functions" and "Algorithm for satisficing solution of problem" sections, our approach for dealing with a multi-objective programming problem with fuzzy number coefficients is presented. In "The stability set of the first kind" section, we determine the stability set of the first kind. "An illustrated example" section is devoted to a numerical example for the sake of illustration. We end up in "Conclusion" section with some concluding remarks along with perspectives for further research in this field. 


\section{Preliminaries}

Fuzzy numbers

Ranking of fuzzy numbers through the comparison of their expected intervals

A fuzzy subset $\tilde{A}$ of the real line $\mathbb{R}$ with membership function $\mu_{\tilde{A}}: \mathbb{R} \rightarrow[0,1]$ is called a fuzzy number if:

(i) $\tilde{A}$ is normal, i.e., there is $x_{0} \in \mathbb{R}$ such that $\mu_{\tilde{A}}\left(x_{0}\right)=1$.

(ii) $\tilde{A}$ is fuzzy convex that is:

$$
\mu_{\tilde{A}}\left(\lambda x_{1}+(1-\lambda) x_{2}\right) \geq \min \left(\mu_{\tilde{A}}\left(x_{1}\right), \mu_{\tilde{A}}\left(x_{2}\right)\right)
$$

for $x_{1}, x_{2} \in \mathbb{R}, \quad \lambda \in[0,1]$.

Jiménez (1996) has proposed a ranking method of fuzzy numbers based on the comparison of their expected intervals. This method is employed in this paper for developing a direct solution approach for the given multi-objective with fuzzy parameters.

The membership function of a fuzzy number $\tilde{A}=\left(\underline{a} ; a_{1} ; a_{2} ; \bar{a}\right)$ can be written as

$$
\mu_{\tilde{A}}(x)= \begin{cases}0, & \forall x \in(-\infty, \underline{a}], \\ f_{A}(x), & \forall x \in\left[\underline{a}, a_{1}\right], \\ 1, & \forall x \in\left[a_{1}, a_{2}\right], \\ g_{A}(x), & \forall x \in\left[a_{2}, \bar{a}\right], \\ 0, & \forall x \in[\bar{a}, \infty) .\end{cases}
$$

To the existence, given the inverse functions $f_{A}^{-1}(x)$ and $g_{A}^{-1}(x)$, it is assumed that $f_{A}(x)$ is continuous and increasing and $g_{A}(x)$ is continuous and decreasing.

The expected interval of a fuzzy number is defined as follows (Jiménez 1996):

$$
E I(\tilde{A})=\left[E_{1}^{\tilde{A}}, E_{2}^{\tilde{A}}\right]=\left[\int_{\vec{a}}^{a_{1}} x \mathrm{~d} f_{A}(x),-\int_{a_{2}}^{\bar{a}} x \mathrm{~d} g_{A}(x)\right] .
$$

Integrating by making change in the variable $\alpha$ to $\alpha=f_{A}(x)$, and $\alpha=g_{A}(x)$ in the first and second integrations, respectively:

$$
E I(\tilde{A})=\left[E_{1}^{\tilde{A}}, E_{2}^{\tilde{A}}\right]=\left[\int_{0}^{1} f_{A}^{-1}(\alpha) \mathrm{d} \alpha,-\int_{0}^{1} g_{A}^{-1}(\alpha) \mathrm{d} \alpha\right] .
$$

If $f_{A}$ and $g_{A}$ are linear, that is if the fuzzy number $\tilde{A}$ is triangular or trapezoidal, its expected interval will be as in (4) (Jiménez 1996). If the fuzzy number $\tilde{A}$ is triangular, then $a_{1}=a_{2}=a$ and can be written as $\tilde{A}=(\underline{a}, a, \bar{a})$.

$$
E I(\tilde{A})=\left[\frac{1}{2}\left(\underline{a}+a_{1}\right), \frac{1}{2}\left(a_{2}+\bar{a}\right)\right]
$$

If there are two fuzzy numbers $\tilde{A}$ and $\tilde{B}$, the expected interval of $\tilde{A}-\tilde{B}$ is

$$
E I(\tilde{A}-\tilde{B})=\left[E_{1}^{\tilde{A}}-E_{2}^{\tilde{B}}, E_{2}^{\tilde{A}}-E_{1}^{\tilde{B}}\right]=E I(\tilde{A})-E I(\tilde{B}) .
$$


According to the ranking method of Jimenez, for any pair of fuzzy numbers $\tilde{A}$ and $\tilde{B}$, the degree in which $\tilde{A}$ is bigger than $\tilde{B}$ is defined as (Jiménez 1996)

$$
\mu(\tilde{A}, \tilde{B})= \begin{cases}0 & \text { if } E_{2}^{\tilde{A}}-E_{1}^{\tilde{B}}<0, \\ \frac{E_{2}^{\tilde{A}}-E_{1}^{\tilde{B}}}{E_{2}^{\tilde{A}}-E_{1}^{\tilde{B}}-\left(E_{1}^{\tilde{A}}-E_{2}^{\tilde{B}}\right)}, & \text { if } 0 \in\left[E_{1}^{\tilde{A}}-E_{2}^{\tilde{B}}, E_{2}^{\tilde{A}}-E_{1}^{\tilde{B}}\right], \\ 1 & \text { if } E_{1}^{\tilde{A}}-E_{2}^{\tilde{B}}>0 .\end{cases}
$$

where $\mu(\tilde{A}, \tilde{B})$ is the degree of preference of $\tilde{A}$ over $\tilde{B}$. When $\mu(\tilde{A}, \tilde{B})=0.5$ it will be said that $\tilde{A}$ and $\tilde{B}$ are equal.

The expected value of a fuzzy number is the half point of its expected interval:

$$
E V(\tilde{A})=\frac{E_{1}^{\tilde{A}}+E_{2}^{\tilde{A}}}{2} .
$$

If the fuzzy number $\tilde{A}$ is triangular or trapezoidal, its expected value will be (Jiménez 1996)

$$
E V(\tilde{A})=\frac{1}{4}\left(\underline{a}+a_{1}+a_{2}+\bar{a}\right) .
$$

Proposition 1 Luhandjula and Rangoaga (2014)

Define

$$
\begin{array}{r}
f_{-}:[0,1] \rightarrow \mathbb{R} \\
\alpha \rightarrow f_{-}(\alpha)=\underline{\tilde{a}}_{\alpha}
\end{array}
$$

and

$$
\begin{aligned}
\bar{f}:[0,1] & \rightarrow \mathbb{R} \\
\alpha \rightarrow \bar{f}(\alpha) & =\overline{\tilde{a}}_{\alpha}
\end{aligned}
$$

then

$$
C_{d}(\tilde{A})=\left[\frac{1}{2}\left(\underline{a}+a_{1}\right), \frac{1}{2}\left(a_{2}+\bar{a}\right)\right] .
$$

\section{Algorithm for finding a rank of a fuzzy number Algorithm 1}

Step 0: Start

Step 1: $\quad \operatorname{Read} \mu_{\tilde{A}}(x)$

Step 2: $\quad$ Define $f(\alpha)=\tilde{a}, \bar{f}(\alpha)=\overline{\tilde{a}}_{\alpha}$,

$\begin{array}{ll}\text { Step 3: } & \text { Find } C_{d}(\tilde{\tilde{A}})=\left[\frac{q}{2}\left(\underline{a}+a_{1}\right), \frac{1}{2}\left(a_{2}+\bar{a}\right)\right] . \\ \text { Step 4: } & \text { Print } C_{d}(\tilde{A})\end{array}$

Step 5: Stop 


\section{Finding a satisfying solution of a multi-objective program with fuzzy objective functions}

\section{Problem formulation}

We consider a mathematical program of the form:

$$
\begin{aligned}
& \operatorname{Min}\left(\tilde{f}_{1}(x), \tilde{f}_{2}(x), \ldots, \tilde{f}_{m}(x)\right) \\
& \text { subject to } x \in X=\left\{x \in \mathbb{R}^{n} \mid g_{j}(x) \leq 0, j=1,2, \ldots, k\right\}
\end{aligned}
$$

where $\tilde{f}_{i}(x), i=(1,2, \ldots, m)$ are a function from $\mathbb{R}^{n}$ to $\mathrm{F}\left(f\left(\mathbb{R}^{n}\right)\right)$ and $g_{j}(j=1, \ldots, k)$ are a function from $\mathbb{R}^{n}$ to $\mathbb{R}$, where $\mathrm{F}\left(f\left(\mathbb{R}^{n}\right)\right)$ is the image of objective function.

This model comes up in several applications including water resource management (Bravo and Gonzalez 2009), production planning problem (Escudero et al. 2009) and power plant maintenance scheduling (Canto 2008).

It is not a loss of generality to focus on the case of deterministic constants. As a matter of fact, the literature is rich of papers addressing the problem of converting fuzzy constraints into crisp ones. See for example Luhandjula (1989) and Bhaskar et al. (2004). The problem (9) is an ill-stated problem due to the presence of several objective functions coupled to fuzziness surrounding involved data.

Therefore, the optimum does not exist for this problem and we have to seek for some satisfying solution. Moreover, existing approaches for the deterministic case, like the weighting method, cannot be applied blindly. They should be tailored to make them suitable for the fuzzy case.

Let us define the following scalarization for fuzzy multi-objective nonlinear programming (FMONLP) problem, using the membership ranking function and a modified crisp model

$$
\operatorname{Min} \operatorname{Max} \rho \sum \tilde{A}_{j}^{l} x_{j}+\left(\sum_{i=1}^{m} \frac{\tilde{f}_{i}(x)}{f_{-i}-\bar{f}_{i}}\right)
$$

subject to

$$
\begin{aligned}
& x \in X=\left\{x \in \mathbb{R}^{n} \mid g_{j}(x) \leq 0\right\}, \quad \ell=1,2, \ldots, q, \\
& \lambda \geq 0, \quad x_{j} \geq 0, \quad i=1, \ldots, m, \quad j=1,2, \ldots, k .
\end{aligned}
$$

where $\rho, f_{-i}=\min \tilde{f}_{i}(x), \bar{f}_{i}=\max \tilde{f}_{i}(x), i=1,2, \ldots, m$. is a sufficiently small positive scalar

The problem (10) can be reformulated to take the following equivalent form (Kassem 2008).

$\operatorname{Min} \lambda$

subject to

$$
\rho \sum \tilde{A}_{j}^{l} x_{j}+\left(\sum_{i=1}^{m} \frac{\tilde{f}_{i}(x)}{f_{-i}-\bar{f}_{i}}\right) f_{-i} \geq \lambda, \quad i=1,2, \ldots, m
$$




$$
\begin{aligned}
& x \in X=\left\{x \in \mathbb{R}^{n} \mid g_{j}(x) \leq 0\right\}, \quad \ell=1,2, \ldots, q, \\
& \lambda \geq 0, \quad x_{j} \geq 0, \quad i=1,2, \ldots, m, \quad j=1,2, \ldots, k .
\end{aligned}
$$

where $\lambda=\operatorname{Max} \rho \sum \tilde{A}_{j}^{l} x_{j}+\left(\sum_{i=1}^{m} \frac{\tilde{f}_{i}(x)}{f_{i}-\bar{f}_{i}}\right), i=1, \ldots, m$.

Moreover, it has not inconvenienced of defuzzification operators that transform a fuzzy number into the real number, leading to a loss of too much information about fuzzy numbers. Furthermore, the ranking number operator is optimal in the sense that it is among of a fuzzy member, that minimizes the fuzzy number.

\section{The stability set of the first kind}

Definition 4.1. Stability Generally, we call the numerical method is stable if the numerical errors which are generated during the solution of discretized equations should not be magnified, i.e., the numerical solution is close to the analytical solution. Here, the stability set of the first kind means that the set of all parameters say $(\rho, \lambda)$ which ensures a solution $(\bar{x}, \bar{\lambda})$ of FMONLP problem is optimal.

In general, a numerical method is stable if the cumulative effect of all errors, including round-off errors, is bounded independent of the mesh points, i.e., the numerical solution is close to the analytical solution. Here, we consider a stability set of the first kind which means that the set of all parameters that ensures a numerical solution of FMONLP problem is optimal.

Definition 4.2 Given a certain $(\rho, \lambda)$ with a corresponding optimal solution $(\bar{x}, \bar{\lambda})$; then the stability set of the first kind of the problem (9) corresponding to this optimal solution is defined by:

$$
S(\bar{x}, \bar{\lambda})=\left\{(\rho, \lambda) \in R^{m+1}:(\bar{x}, \bar{\lambda}) \text { is an optimal solution of FMONLP problem }\right\} .
$$

On account of Rockafellar (1967), we can consider the problem (9) is stable of the first kind and hence problem (10) is also stable.

From the stability of the first kind of the problem (10), there exists $\rho, \lambda, \tilde{A} \geq 0, u \in R^{m}, u \geq 0$, and $v \in R^{r}, v \geq 0$ such that the Kuhn-Tucker conditions of problem (10) take the form:

$$
\begin{aligned}
& \sum_{i=1}^{m} u_{i} \frac{\partial f_{i}}{\partial x_{\alpha}}+\rho \sum_{i=1}^{m} u_{i} \tilde{A}_{i}^{\alpha} \frac{\partial f_{i}}{\partial x_{\alpha}}+\sum_{j \in J} v_{j} \frac{\partial g_{j}}{\partial x_{\alpha}}=0, \quad \alpha=1,2, \ldots, n, \\
& \sum_{i=1}^{m} u_{i}=1, \\
& \lambda-\rho \sum_{i=1}^{m} \tilde{A}_{i}^{\alpha} \frac{\partial f_{i}}{\partial x_{\alpha}}+\left(\sum_{i=1}^{m} \frac{\tilde{f}_{i}(x)}{f_{-i}-\bar{f}_{i}}\right) f_{-i} \leq 0, \quad i=1,2, \ldots, m,
\end{aligned}
$$




$$
\begin{aligned}
& v_{j} g_{j}(\bar{x})=0, \quad j \in J \subset\{1,2, \ldots, r\} \\
& u_{i}\left\{\lambda-\rho \sum_{i=1}^{m} \tilde{A}_{i}^{\alpha} x_{i}+\left(\sum_{i=1}^{m} \frac{\tilde{f}_{i}(x)}{f_{-i}-\bar{f}_{i}}\right) f_{-i}\right\} \leq 0, \quad i=1,2, \ldots, m, \\
& u_{i} \geq 0, \quad i=1,2, \ldots, m, \\
& v_{j} \geq 0, \quad j \in J, \\
& v_{j}=0, \quad j \notin J .
\end{aligned}
$$

The determination of the stability set of the first kind $S(\bar{x}, \bar{\lambda})$ depends only on whether any of the variables $u_{i}, i=1,2, \ldots, m$, and any of the variables $v_{j}=0, j \in J \subset\{1,2, \ldots, r\}$ which solve Eqs. (12), (13), and (17), then in order that the other Kuhn-Tucker conditions (14) and (16) are satisfied, we must have

$$
\begin{aligned}
& \lambda_{i}=\rho \sum_{i=1}^{m} \tilde{A}_{i}^{\alpha} \frac{\partial f_{i}}{\partial x_{\alpha}}+\left(\sum_{i=1}^{m} \frac{\tilde{f}_{i}(x)}{f_{-i}-\bar{f}_{i}}\right) f_{-i}, \quad i \notin I ; \\
& \lambda_{i} \leq \rho \sum_{i=1}^{m} \tilde{A}_{i}^{\alpha} \frac{\partial f_{i}}{\partial x_{\alpha}}+\left(\sum_{i=1}^{m} \frac{\tilde{f}_{i}(x)}{f_{-i}-\bar{f}_{i}}\right) f_{-i}, \quad i \in I .
\end{aligned}
$$

Let

$D=\left\{I \mid u_{i}=0 ; i \in I, u_{i}>0, i \notin I\right.$ solve (12), (13), (17-20) $\}$

and

$$
\begin{aligned}
S(\bar{x}, \bar{\lambda})= & \left\{(\rho, \lambda) \in R^{m+1} \mid \lambda_{i}=\rho \sum_{i=1}^{m} \tilde{A}_{i}^{\alpha} x_{i}+\left(\sum_{i=1}^{m} \frac{\tilde{f}_{i}(x)}{f_{-i}-\bar{f}_{i}}\right) f_{-i}, \quad i \notin I,\right. \\
& \text { and } \\
& \left.\lambda_{i} \leq \rho \sum_{i=1}^{m} \tilde{A}_{i}^{\alpha} x_{i}+\left(\sum_{i=1}^{m} \frac{\tilde{f}_{i}(x)}{f_{-i}-\bar{f}_{i}}\right) f_{-i}, i \in I\right\} .
\end{aligned}
$$

Then, it is clear that

$$
S(\bar{x}, \bar{\lambda})=\bigcup_{I \in D} S_{I}(\bar{x}, \bar{\lambda})
$$




\section{Algorithm for satisficing solution of problem (9)}

From the discussion, we can derive the following algorithm for finding a satisficing solution of problem (9)

\section{Algorithm 2}

Step 0: Input $m, k$

Input objective function: $\tilde{f}_{1}(x), \tilde{f}_{2}(x), \ldots, \tilde{f}_{m}(x)$

Input constraint function: $g_{1}(x), \ldots, g_{j}(x)$.

Step 1: Finding fuzzy number of $\tilde{f}_{1}(x), \tilde{f}_{2}(x), \ldots, \tilde{f}_{m}(x)$.

(a) Put $l=1$

(b) Repeat until break

If $l \leq m$ go to Algorithm 1 with input $\tilde{f}_{l}(x)$. Find $f_{-l}(x)$ and $\bar{f}_{l}(x)$. Else, break and go to (c).

Step 2: Finding a satisfying solution of problem (9) by solving problem (21)

$\operatorname{Min} \lambda$

subject to

$$
\begin{aligned}
& \rho \sum \tilde{A}_{j}^{l} x_{j}+\left(\sum_{i=1}^{m} \frac{\tilde{f}_{i}(x)}{f_{-i}-\bar{f}_{i}}\right) f_{-i} \geq \lambda, \quad i=1,2, \ldots, m \\
& x \in X=\left\{x \in \mathbb{R}^{n} \mid g_{j}(x) \leq 0\right\}, \quad \ell=1,2, \ldots, q, \\
& \lambda \geq 0, \quad x_{j} \geq 0, \quad i=1,2, \ldots, m, \quad j=1,2, \ldots, k .
\end{aligned}
$$

If problem (21) has solution $x^{*}$, then go to $(\mathrm{d})$

(c) If such problem (21) cannot be found go to (f)

Print " $x$ " is a satisficing solution of problem (9)".

(d)Determine the stability set of the first kind, $S(\bar{x}, \bar{\lambda})$, corresponding to this solution.

(e) Print: "There is no satisficing solution of problem (9)".

(f) Stop.

\section{An illustrated example}

In this section, some calculations are carried out to illustrate the efficiency of the proposed method. Here, we consider only one of these examples for brevity:

Consider the following multi-objective program:

$$
\begin{array}{r}
\min \tilde{A}_{1}^{1} x_{1}+\tilde{A}_{2}^{1} x_{2}, \tilde{A}_{1}^{2} x_{1}+\tilde{A}_{2}^{2} x_{2} \\
\text { subject to } x_{1}+x_{2} \geq 6, \\
2 x_{1}+x_{2} \geq 9
\end{array}
$$


where $\tilde{A}_{j}^{\ell},(\ell=1,2 ; j=1,2)$ are fuzzy numbers the membership function of which are given below:

$$
\begin{aligned}
& \mu_{\tilde{A}_{1}^{1}}(x)= \begin{cases}2 x-1 & \text { for } x \in[0.5,1] \\
-2 x+3 & \text { for } x \in[1,1.5] \\
0 & \text { otherwise }\end{cases} \\
& \mu_{\tilde{A}_{2}^{1}}(x)= \begin{cases}5 x-9 & \text { for } x \in[1.8,2] \\
-x+3 & \text { for } x \in[2,3] \\
0 & \text { otherwise }\end{cases} \\
& \mu_{\tilde{A}_{1}^{2}}(x)= \begin{cases}\frac{1}{2} x-1 & \text { for } x \in[0,2] \\
-x+3 & \text { for } x \in[2,3] \\
0 & \text { otherwise }\end{cases} \\
& \mu_{\tilde{A}_{2}^{2}}(x)= \begin{cases}x & \text { for } x \in[0,1] \\
-\frac{1}{2} x+\frac{3}{2} & \text { for } x \in[1,3] . \\
0 & \text { otherwise }\end{cases}
\end{aligned}
$$

Using Algorithm 1, we obtain the fuzzy numbers as follows:

$$
\begin{aligned}
& C_{d}\left[\tilde{A}_{1}^{1}\right]=\left[\frac{1}{2}(0.5+1), \frac{1}{2}(1+1.5)\right]=[0.75,1.25] \\
& C_{d}\left[\tilde{A}_{2}^{1}\right]=\left[\frac{1}{2}(1.8+2), \frac{1}{2}(2+3)\right]=[1.9,2.5] \\
& C_{d}\left[\tilde{A}_{1}^{2}\right]=\left[\frac{1}{2}(0+2), \frac{1}{2}(2+3)\right]=[1,2.5] \\
& C_{d}\left[\tilde{A}_{2}^{2}\right]=\left[\frac{1}{2}(0+1), \frac{1}{2}(1+3)\right]=[0.5,2] .
\end{aligned}
$$

Using Algorithm 1 given by Luhandjula and Rangoaga (2014),

$$
\begin{aligned}
& C_{d}\left[\tilde{A}_{1}^{1}\right]=\left[\frac{1}{2} \int_{0}^{1}(\alpha+1) d \alpha, \frac{1}{2} \int_{0}^{1}(3-\alpha) d \alpha\right]=[0.75,1.25 \\
& C_{d}\left[\tilde{A}_{2}^{1}\right]=\left[\frac{1}{5} \int_{0}^{1}(\alpha+9) d \alpha, \int_{0}^{1}(3-\alpha) d \alpha\right]=[1.9,2.5] \\
& C_{d}\left[\tilde{A}_{1}^{2}\right]=\left[\int_{0}^{1} 2 \alpha d \alpha, \int_{0}^{1}(3-\alpha) d \alpha\right]=[1,2.5] \\
& C_{d}\left[\tilde{A}_{2}^{2}\right]=\left[\int_{0}^{1} \alpha d \alpha, \int_{0}^{1}(3-2 \alpha) d \alpha\right]=[0.5,2] .
\end{aligned}
$$


Obtaining the system using Algorithm 2, we have

$\operatorname{Min} \lambda$

subject to

$$
\begin{aligned}
& 0.75 x_{1}+1.9 x_{2}+\left(\frac{0.75 x_{1}+1.9 x_{2}}{12}+\frac{x_{1}+0.5 x_{2}}{18}\right) \lambda \geq 4.5 \\
& 1.25 x_{1}+2.5 x_{2}+\left(\frac{1.25 x_{1}+2.5 x_{2}}{12}+\frac{2.5 x_{1}+2 x_{2}}{18}\right) \lambda \geq 13.5 \\
& x_{1}+0.5 x_{2}+\left(\frac{0.75 x_{1}+1.9 x_{2}}{12}+\frac{x_{1}+0.5 x_{2}}{18}\right) \lambda \geq 4.5 \\
& 2.5 x_{1}+2 x_{2}+\left(\frac{1.25 x_{1}+2.5 x_{2}}{12}+\frac{2.5 x_{1}+2 x_{2}}{18}\right) \lambda \geq 13.5 \\
& x_{1}+x_{2} \geq 6 \\
& 2 x_{1}+x_{2} \geq 9
\end{aligned}
$$

The solution of the system $(23)$ is $x^{*}=(3,3)$, and the corresponding stability set of the first kind is

$$
S(\bar{x}, \bar{\lambda})=\left\{\left(6.9 \lambda_{2}-7.5 \lambda_{1}\right) \rho=0.49,0.484 \geq\left(6.9 \lambda_{1}+7.5 \lambda_{2}\right) \rho\right\} .
$$

On the other hand, using Algorithm 2 given by Luhandjula and Rangoaga (2014), we get the system

$$
\begin{array}{ll}
\lambda_{1}\left[\left(\begin{array}{r}
0.75 \\
1.9
\end{array}\right)+\left(\begin{array}{r}
1.25 \\
2.5
\end{array}\right)\right]+\lambda_{2}\left(\begin{array}{r}
1 \\
0.5
\end{array}\right)+\left(\begin{array}{r}
2.5 \\
2
\end{array}\right)+\mu_{1}\left(\begin{array}{l}
-1 \\
-2
\end{array}\right)+\mu_{2} & =0, \\
\mu_{1}\left(-x_{1}-x_{2}+6\right) & =0, \\
\mu_{2}\left(-2 x_{1}-x_{2}+9\right) & =0, \\
\lambda_{1}>0, \lambda_{2}>0, \mu_{1} \geq 0, \mu_{2} & \geq 0 .
\end{array}
$$

The solution of the system $(23)$ is $x^{*}=(3,3)$.

\section{Conclusion}

Some numerical calculations have been carried out on different examples and the CPU time taken by the proposed method and Luhandjula and Rangoaga (2014) method is compared. The results show that proposed method has less CPU time around 75\% than Luhandjula and Rangoaga (2014) method. Moreover, the proposed method which depends on ranking of fuzzy numbers shows that it is much easier and efficient than the method mentioned in Luhandjula and Rangoaga (2014); while in the last method, it depends on obtaining nearest interval approximation and computing some integrations.

An approach that strikes a balance between effectiveness and efficiency while coping with multi-objective programming problems with fuzzy objective functions is 
introduced. Algorithms are given in a way to make the transition from theory to practice easy and can be also carried out.

Indeed, the considered approach is to extend the approaches described in Joubert and Luhandjula (2010) and Skawa and Yauchi (1999), where randomness and fuzziness are under one roof in an optimization framework.

Finally, for further research, analysis of fuzzy multi-objective programming method can be successfully applied to serve the decision makers and to push forward a decision support system (DSS) (Eom and Kim 2006).

\section{Authors' contributions}

MAK and AMT proposed the idea. AMT and NME implemented the calculations and helped in drafting and writing of this manuscript. All authors read and approved the final manuscript.

\section{Author details}

${ }^{1}$ Mathematics Department, Faculty of Science, Damietta University, Damietta, Egypt. ${ }^{2}$ Mathematics Department, Faculty of Science, Tanta University, Tanta, Egypt.

\section{Competing interests}

The authors declare that they have no competing interests.

Received: 29 September 2016 Accepted: 22 December 2016

Published online: 05 January 2017

\section{References}

Adeyefa A, Luhandjula M (2011) Multiobjective stochastic linear programming: an overview. Am J Oper Res 1(4):03-213

Bhaskar PG, Krishnan, Sundsrarajan R (2004) A fuzzy mathematical programming approach for cross-sell optimization in retail and banking. J Oper Res Soc 60:717-727

Bravo M, Gonzalez I (2009) Applying stochastic goal programming: a case study on water use planning. Eur J Oper Res 196:1123-1129

Canto S (2008) Application of Benders decomposition to power plant preventive maintenance scheduling. Eur J Oper Res 184:759-777

Dubois D, Prade H (1980) Fuzzy sets and systems, theory and applications. Academic Press, New York

Eom S, Kim E (2006) A survey of decision support system applications. J Oper Res Soc 57:1264-1278

Escudero LF, Kamesam PV, King AJ, Wets RJB (2009) Production planning via scenario modelling. Ann Oper Res 43:309-335

Inuiguchi M, Ramik J (2000) Possibilistic linear programming: a brief review of fuzzy mathematical programming and comparison with stochastic programming portfolio selection problem. Fuzzy Sets Syst 111:3-28

Jiménez M (1996) Ranking fuzzy numbers through the comparison of its expected intervals. Int J Uncertain Fuzziness Knowl Based Syst 4:379-388

Jiménez M, Rodri'guez M, Arenas M, Bilbao A (2000) Solving a possibilistic linear program through compromise programming. Mathw Soft Comput 7:175-184

Jiménez M, Arenas M, Bilbao Rodri'guez MV (2007) Linear programming with fuzzy parameters: an interactive method resolution. Eur J Oper Res 177(1):1599-1609

Joubert JW, Luhandjula MK (2010) On some optimisation models in a fuzzystochastic environment. Eur J Oper Res 207(3):1433-1441

Kall P (1976) Stochastic linear programming. Springer, Berlin

Kampempe J, Luhandjula M (2012) Chance-constrained approaches for multiobjective stochastic linear programming problems. Am J Oper Res 2(4):519-526

Kassem MA (2008) Stability achievement scalarization function for multiobjective nonlinear programming problems. Appl Math Model 32:1044-1055

Luhandjula MK (1989) Fuzzy optimization: an appraisal. Fuzzy Sets Syst 30:257-282

Luhandjula MK (2007) Fuzzy mathematical programming: theory, applications and extensions. J Uncertain Syst 1:123-135

Luhandjula MK, Rangoaga MJ (2014) An approach for solving a fuzzy multi objective programming problem. Eur J Oper Res 232:249-255

Rockafellar R (1967) Duality and stability in extremal problems involving convex functions. Pac J Math 21:167-181

Rommelfanger H (1996) Fuzzy linear programming and applications. Eur J Oper Res 92:512-528

Skawa M, Yauchi K (1999) An interactive fuzzy satisficing method for multi objective nonconvex programming through floating point genetic algorithms. Eur J Oper Res 117:113-124

Vajda S (1972) Probablistic programming. Academic Press, New York

Zadeh LA (1978) Fuzzy sets as a basis for a theory of possibility. Fuzzy Sets Syst 1:61-72

Zimmermann HJ (1983) Fuzzy mathematical programming. Comput Oper Res 10:291-298

Zimmermann HJ (1987) Fuzzy set, decision making and expert systems. Kluwer Academic Publisher, Dordrecht 